\title{
Superbubbles in Dwarf Galaxies: Blown Out or Blown Away?
}

\author{
Mordecai-Mark Mac Low ${ }^{1}$ and Andrea Ferrara ${ }^{2}$ \\ 1 MPI für Astronomie, Königstuhl 17, D-69117 Heidelberg, Germany \\ 2 Osservatorio Astrofisico di Arcetri, Largo Enrico Fermi 5, I-50125 Firenze, Italy
}

\begin{abstract}
We numerically model the impact of superbubbles produced by starbursts on the gas in dwarf galaxies, using realistic gravitational potentials including the contributions from dark matter haloes, and galaxy radii based on empirical laws. We find that the metal-rich gas is very efficiently ejected in many cases, contradicting previous results; the ambient gas is far more difficult to eject. X-ray emitting plumes can last more than $10^{8} \mathrm{yr}$ after the end of the active starburst.
\end{abstract}

\section{Introduction}

One of the most extreme examples of bubble dynamics occurs in dwarf galaxies undergoing starbursts. The stellar winds and supernovae from massive stars produce huge shock waves that can blow out of the dwarf's interstellar medium, and might even be able to blow away all the gas from the disk (e.g. De Young \& Heckman 1995), raising its radial velocity above the local escape velocity from the gravitational potential. The rotation curves of dwarf galaxies suggest that their gravitational potentials are dominated by dark matter (Persic, Salucci \& Stel 1996). There is now observational evidence in support of the existence of outflows from dwarf galaxies including NGC 1705 (Meurer et al.1992), I Zwicky 18 (Martin 1996), NGC 1569 (Della Ceca et al.1996) and a number of others in a survey by Marlowe et al.(1995). In this paper, we numerically model the effect of nuclear starbursts on dwarf galaxies, including the effect of dark matter for the first time.

\section{Models}

We model a dwarf galaxy as a system made of a gaseous and stellar (i.e. visible) disk of mass $M_{d}$ and a dark halo of mass $M_{h}$. We assume that the gaseous disk has constant surface density, motivated by observations of our Galaxy. Persic, Salucci \& Stel (1996) find that the dark-to-visible mass ratio $\phi=M_{h} / M_{d}$ is a function of the galactic mass, $\phi \simeq 34.7 M_{d, 7}^{-0.29}$, where $M_{d, 7}=$ $M_{d} / 10^{7} M_{\odot}$. We assume that the halo can be approximated by a softened isothermal sphere, with scale radius and central density dependent on total mass (Burkert 1995). From these assumptions, we can derive the gravitational potential of the halo, and compute the exact, hydrostatic equilibrium of the 
Table 1. Efficiencies

\begin{tabular}{l|lll|lll} 
& \multicolumn{3}{|c}{ Mass Eject. Eff. } & \multicolumn{3}{c}{ Metal Ret. Eff. } \\
\hline Lum & \multicolumn{3}{|c}{ Mass (M $\left.\mathrm{M}_{\odot}\right)$} & \multicolumn{3}{c}{ Mass (M $\odot)$} \\
$\left(10^{38} \mathrm{erg} \mathrm{s}^{-1}\right)$ & $10^{7}$ & $10^{8}$ & $10^{9}$ & $10^{7}$ & $10^{8}$ & $10^{9}$ \\
\hline 0.1 & $4.6(-3)$ & 0.0 & 0.0 & $4.1(-1)$ & 1.0 & 1.0 \\
1.0 & $1.4(-2)$ & $6.5(-4)$ & $1.0(-6)$ & $2.7(-3)$ & $4.0(-1)$ & $9.7(-1)$ \\
10.0 & $6.8(-2)$ & $4.8(-3)$ & $1.3(-4)$ & $4.9(-3)$ & $1.7(-2)$ & $4.5(-1)$
\end{tabular}

gas in this potential. From an empirical fit to a sample of dwarf galaxies Ferrara \& Tolstoy (in preparation) find that the radius-HI mass relation is well approximated by the law $R \simeq(3 \mathrm{kpc}) M_{g, 7}^{0.338}$, so we cut off our gas distributions at this radius.

For our numerical models, we use ZEUS-3D ${ }^{1}$, a second-order, Eulerian, astrophysical gas dynamics code (Stone \& Norman 1992), including equilibrium radiative cooling (MacDonald \& Bailey 1981), and a tracer field in order to maintain a sharp interface at the edge of the hot bubble (using the Yabe-Xiao transform on the tracer function [Yabe \& Xiao 1993]).

We set up the gas at a constant sound speed of $10 \mathrm{~km} / \mathrm{s}$, with adiabatic index $\gamma=5 / 3$, and minimum density $n_{\mathrm{IGM}}=6.4 \times 10^{-8} \mathrm{~cm}^{-3}$. We take the central energy source to be a constant luminosity wind driven by a thermal energy source. We use 2D, azimuthally-symmetric grids centered on the center of the galaxies, with reflecting boundary conditions along the symmetry axis and along the galaxy mid-plane, and outflow boundary conditions on the other two axes, and having a resolution of 20 zones per scale height, with the outer regions covered by a grid ratiod at 1.03 per zone.

\section{Results}

In Figure 1 we show the density distribution of every model in the parameter study. We see the holes in the plane of the galaxy recollapsing under the influence of gravity and the pressure of the disk gas. At the same time, the hot bubbles rising above the disks expand out into the surrounding intergalactic medium, leaving most of the swept-up shell material behind.

We can directly compute the efficiency of mass ejection and retention of metal-enriched gas from our models. In Table 1 we show the fraction of mass accelerated beyond escape velocity. Even in our most extreme model, with a supernova every 30,000 years in a galaxy of mass $10^{7} \mathrm{M}_{\odot}$, less than $10 \%$ of the mass is ejected, and the efficiency drops rapidly from there.

${ }^{1}$ Developed by the Laboratory for Computational Astrophysics at the NCSA, and available for community use by registration at lca@ncsa.uiuc.edu 


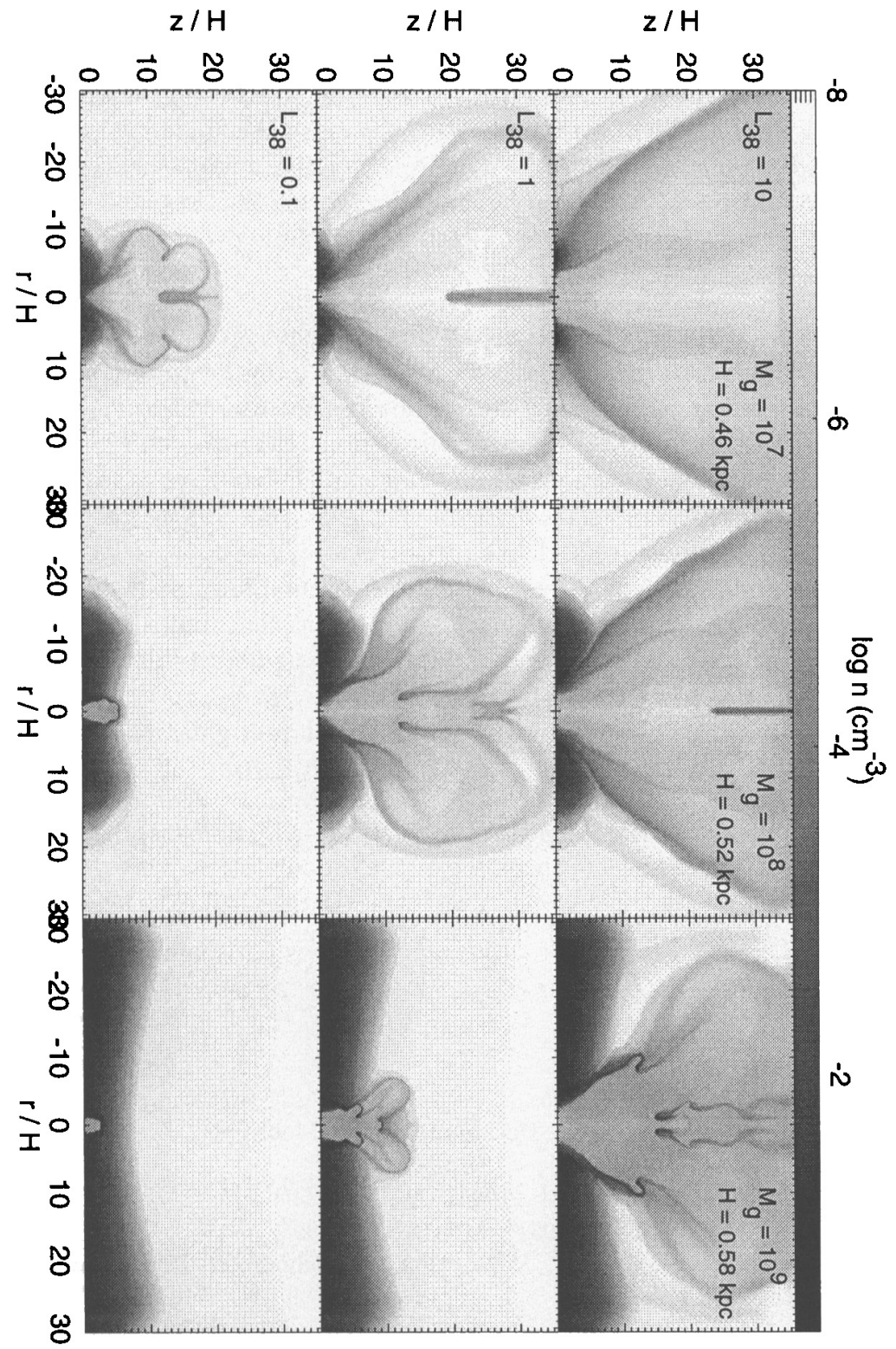

Fig. 1. Density distributions of models of galaxies with the given masses in solar masses, containing starbursts of the given mechanical luminosities in units of $10^{38}$ $\mathrm{erg} \mathrm{s}^{-1}$ at a time of $100 \mathrm{Myr}$, some $50 \mathrm{Myr}$ after the end of energy input from the starbursts, with the values of density given by the colorbar at the top of the figure. Lengths are given in terms of the best-fitting exponential scale height for each model, with values quoted in the figure. Luminosities are equivalent to supernovae every 30,000 to 3 million $\mathrm{yr}$. 
Using our tracer field, we can also find the fraction of metal-enriched gas retained for each model (Table 1). In the more massive galaxies and at lower supernova rates, the metal enriched gas is retained in the gravitational well of the dark halo, and will eventually fall back on to the galaxy, while at lower masses and higher luminosities, virtually all of the metals escape the grasp of the halo and travel freely into surrounding intergalactic space.

Finally, we discuss some predictions of observable properties that we can draw from our models:

- Hot gas is well separated from the dense filaments observed in $\mathrm{H} \alpha$.

- These filaments are not shock-heated; they may be photoionized from OB stars below, and so may show photoionized line ratios.

- X-ray emission may be strongest in regions close to cooler, dense filaments, where mass evaporates into hot gas. Thus, observed X-ray emission may come from relatively thin sheets of evaporating gas, far out of ionization equilibrium, and so emitting strongly.

- Blowouts can carry field lines, so more extreme blowouts from low-mass, high-luminosity galaxies will have larger radio-continuum haloes.

- Highly-enriched gas far from a galaxy might be detectable in absorption against background QSO's.

- These ideas may also apply to starbursts in larger galaxies, as described by Suchkov et al. (1994).

Acknowledgements. We are grateful to E. Tolstoy, D. Bomans, R.-J. Dettmar, and G. Golla for discussions of the observations. Computations were performed at the Rechenzentrum Garching of the Max-Planck-Gesellschaft. We each thank the other's institute for hospitality during work on this paper.

\section{References}

Burkert, A. (1995): ApJ 447, L25

Della Ceca, R., Griffiths, R. E., Heckman, T. M., MacKenty, J. W. (1996): ApJ 469,662

De Young, D. S., Heckman, T. M. (1994): ApJ 431, 598

MacDonald, J., Bailey, M. E. (1981): MNRAS 197, 995

Marlowe, A. T., Heckman, T. M., Wyse, R. F. G., Schommer, R. (1995): ApJ 438, 563

Martin, C. (1996): ApJ 465, 680

Meurer, G. R., Freeman, K. C., Dopita, M. A., Cacciari, C. (1992): AJ 103, 60

Persic, M., Salucci, P., Stel, F. (1996): MNRAS 281, 27

Stone, J. M., Norman, M. L. (1992): ApJS 80, 753

Suchkov, A. A., Balsara, D. S., Heckman, T. M., Leitherer, C. (1994): ApJ 430, 511

Yabe, T., Xiao, F. (1993): J. Phys. Soc. Japan 62, 2537 